\title{
Tracking with Range-Only Measurements Using a Network of Wireless Sensors
}

\author{
Evangelos B. Mazomenos, Jeffrey S. Reeve, Neil M. White \\ School of Electronics and Computer Science \\ University of Southampton, \\ Southampton, U.K. \\ \{ebm07r, jsr, nmw\}@ecs.soton.ac.uk
}

\begin{abstract}
In this paper we propose a tracking system for wireless sensor networks, which operates on accumulated ranging data from a number of anchor sensor nodes in order to infer the trace and other kinematic characteristics of a mobile target. The nonlinear nature of the "tracking with range-only measurements" problem yields significant challenges. In the proposed system, this problem is modeled as a discrete-time state estimation problem. To achieve tracking of manoeuvring targets, adaptive estimation algorithms and a multi-modal approach to describe the development of the target's state in time are utilized. To solve the estimation problem we employ a Particle Filter based algorithm. We present simulation results, which demonstrate the ability of the proposed system not only to effectively track manoeuvring targets but also to perform accurately under noisy conditions.
\end{abstract}

Index Terms-Wireless Sensor Networks, Tracking, Particle Filters

\section{Motivation}

Wireless Sensor Networks (WSNs) have received and continue to receive significant research interest. The flexibility they offer, resulted in an escalating research effort for employing this novel technology in a number of applications. WSNs provide a new approach to interact with the physical world through the amount of information they collect. As research in minimizing the size and increasing the available computational power in WSNs continues, so will research on possible solutions they can offer in a number of existing and challenging problems. One of the areas where WSNs play a key role is in tracking mobile objects. The ability of WSNs to obtain a vast amount of information in a distributive manner renders them suitable for tracking applications [1]. Locating personnel in industrial infrastructures, wildlife monitoring and vehicle tracking are some examples where tracking of ground targets is desirable. WSNs offer a great alternative to existing tracking technologies such as cameras, micro-RADARS or GPS based systems.

This paper discusses a tracking system intended for deployment in WSNs. Two novel, Particle Filter (PF) inspired, tracking algorithms were designed to achieve online tracking of mobile targets based on a batch of range measurements provided from the wireless sensors. In general, the target's dynamics are inferred by processing a specific modality associated with the target's kinematic variables. The proposed system utilizes range measurements between the target and a number of anchor nodes to infer the desired kinematic characteristics of the target. Other such modalities that can be exploited in tracking systems are bearing and velocity. However, the acquisition of such modalities requires additional hardware like RADARS to be attached to the nodes and additional energy and process power to be consumed. On the other hand, ample ranging estimation between wireless nodes can be achieved using a variety of techniques which are relatively energy efficient and do not require any additional hardware. Examples include Received Signal Strength Indication (RSSI) [2] and Time of Flight (ToF) ranging schemes [3].

The major challenge of this work is twofold. Since WSNs nodes are devices with limited energy supply and processing power, the range estimates acquired by the system will inherently contain significant amount of noise. Subsequently, robustness becomes a necessity. Moreover the system is intended to be as generic as possible. Generic "in terms of a tracking system" means being able to track targets moving at a range of speeds and also include support for manoeuvring targets, which is the case in most real-world scenarios.

The remainder of the paper is organized as follows. The next section discusses similar work carried out in the area. In Section III an overview of the proposed tracking system is provided, followed by the formulation of the tracking problem in mathematical terms. The developed algorithms are analyzed in Section V and simulations evaluate the system's performance in the sequel. Concluding remarks and future work are discussed in the final section of this paper.

\section{RELATED WORK}

Locationing and tracking of ground targets using WSNs has long been an active area of research [4], [5]. A number of research approaches stem from military surveillance scenarios [6], [7], [8], [9]. The focus of such systems is to effectively monitor an area and detect intruders breaching the perimeter coverage. Another important operation, is to successfully classify the intruding target to one of the categories considered. A soldier tracking system is presented by Chen et. al [10]. In this system, a two stage procedure is employed to refine PIR sensor reports, produced by a dense, large-scale WSN, and estimate the target's position [11]. The aforementioned systems, focus primarily on the intruder detection mission they are intended to. Due to the fact that WSNs are ideal for 
positioning and tracking problems significant research effort in the WSN community was devoted to develop locationing and tracking algorithms for WSNs [12]. A collaborative processing scheme for tracking with WSNs is presented in [13], [14]. An algorithm to estimate the number of multiple present targets using a wireless network of PIR sensors is presented in [15]. PIR sensors are also considered for tracking by Shrivastava $e t$. al. [16]. The above approaches although provide significant results consider a relatively simple motion model. In addition, PIR sensors can provide support for detection schemes, however the lack of information related to the target's kinematics makes them unsuitable, particularly for tracking manoeuvring targets. Another disadvantage of PIR sensors is that good accuracy requires a dense large-scale deployment of sensors. A different approach is followed by Zhong et. al., as they propose the use of node sequences to perform tracking in WSNs [17].

Bayesian Estimation theory and particularly Kalman Filters provide the background of modern tracking systems [18]. Hence, they are extensively investigated for tracking in WSNs. Kusy et. al. propose a Kalman Filter derivative for locating mobile nodes based on range estimates using RF-Doppler shifts [19]. However, the inherent inability of Kalman Filters (KF) to deal with nonlinear systems, resulted in a number of alternative approaches like $\mathrm{PF}$ to be investigated in order to provide sufficient support for nonlinear systems [20]. PF are a class of recursive Bayesian Estimation techniques inspired from Monte Carlo Integration methods [21]. PF have been extensively investigated in areas like navigation and tracking producing promising results [20], [22]. In the WSNs community, a number of approaches for applying PF in tracking with WSN has been presented. Coates $e t$. al propose a distributed PF tracking algorithm for WSNs [23], [24]. Borkar et.al consider a network of Direction of Arrival (DOA) sensors and Range-Doppler sensors and a PF algorithm performs the estimation [25]. A distributed PF for tracking in sensor networks is also analyzed in [26], where acoustic sensors are proposed. Recently Ma et.al combine a PF and a Probabilistic Data Association Filter to form a hybrid algorithm intended for tracking in WSNs [27]. These approaches either consider only a linear (constant velocity) model to describe the target's kinematics [26], [27], [25], or consider two or more types of available measurements to the system (range, bearing and velocity) in order to provide support for manoeuvring targets. The system, proposed in this paper operates on range-only measurements both for non-manoeuvring (linear state modelling) and manoeuvring targets (nonlinear state modelling). By utilizing ranging estimates from a number of anchor nodes, support for manoeuvring targets is provided, without the need to add power consuming bearing sensors to the available hardware.

\section{SYSTEM OVERVIEW}

The proposed system performs tracking of ground mobile targets based on range estimations from a number of anchor nodes, positioned in known locations. Knowing the location of anchor nodes can be achieved either by pre-defined positioning of the anchor nodes or with ad-hoc deployment and selflocationing. Self-locationing schemes in WSNs is an active research area [3], [28]. A deployment of our proposed system is illustrated in Figure 1. For ground targets at least three anchor nodes must provide range measurements. A central node receives information from the anchor nodes and employs the tracking algorithm. As mentioned previously two widely known techniques able to provide accurate-enough ranging are RSSI and ToF. These approaches require that an RF transceiver or an additional node, to be strapped to the target, to enable communication with the anchor nodes. By employing ranging techniques, an estimation of the range between the mobile node strapped to the target and the static anchor nodes is produced.

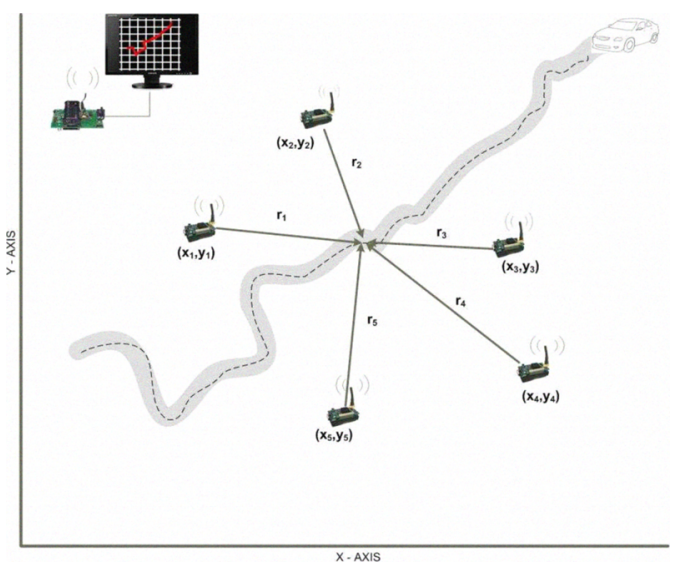

Fig. 1. Tracking System Overview

\section{PROBLEM FormULATION}

In order to best describe the target's movement and since only range measurements are considered, we model our system using a nonlinear discrete-time state-space approach. We provide two options for modeling the state evolution in time, a single-modal and a multi-modal approach. We opt to use a multi-modal approach in order to successfully capture the development of the state vector in time when manoeuvring targets are considered. Prior knowledge regarding the target's movement pattern, points on which approach will better describe the dynamics of the target. Although additional complexity is added to the system when the multi-modal state equation is in use, the application demands on accuracy as well as the available energy and computational power will determine which of these models shall be used, to achieve the optimum trade-off between accurate performance and energy consumption.

Tracking is conducted for a defined amount of time denoted as $T$. The sampling period $T_{s}$ defines the number of sampling steps. At each time step $k$ the state vector comprises of the planar coordinates and two axis velocity of the mobile object. Thus:

$$
\mathbf{x}=\left[\begin{array}{llll}
x & y & v_{x} & v_{y}
\end{array}\right]^{T} \text { ( } \mathrm{T} \text { here denotes transpose) }
$$




\section{A. Measurement model}

A number of $N_{s}$ anchor nodes provide range estimates of the target's two-dimensional Euclidean distance to them; hence the measurements vector $\mathbf{z}_{n, k}$ at time step $k$ is formed as :

$$
\mathbf{z}_{k}=\left[\begin{array}{c}
\sqrt{\left(y_{k}-y_{1}\right)^{2}+\left(x_{k}-x_{1}\right)^{2}} \\
\sqrt{\left(y_{k}-y_{2}\right)^{2}+\left(x_{k}-x_{2}\right)^{2}} \\
\sqrt{\left(y_{k}-y_{3}\right)^{2}+\left(x_{k}-x_{3}\right)^{2}} \\
\vdots \\
\sqrt{\left(y_{k}-y_{N_{s}}\right)^{2}+\left(x_{k}-x_{N_{s}}\right)^{2}}
\end{array}\right]+\mathbf{v}_{k}
$$

- where time index $k$ : is discrete: $k=1,2, \ldots, K$

- $\mathbf{v}_{k}$ : is a $N_{s} \times 1$ independent and identically distributed (i.i.d) measurements noise vector sampled from a known distribution

\section{B. State Evolution}

Two approaches (single-modal, multi-modal) are used to model the development of the state in time. The state vector evolves in time according to the following equation.

$$
\mathbf{x}_{k}=\mathbf{F} \mathbf{x}_{k-1}+\boldsymbol{\Gamma} \mathbf{w}_{k-1}
$$

- matrix $\Gamma$ is,

$$
\Gamma=\left[\begin{array}{cc}
T_{s}^{2} / 2 & 0 \\
0 & T_{s}^{2} / 2 \\
T_{s} & 0 \\
0 & T_{s}
\end{array}\right]
$$

- $T_{s}$ is the sampling period,

- $\mathbf{w}_{k-1}$ is a $2 \times 1$ i.i.d process noise vector sampled from a known distribution which represents any mismodeling effects or disturbances in the state equation

- $\mathbf{x}_{k}$ is the state vector, defined in Eq. 1

Matrix $\mathbf{F}$ is defined in two ways:

1) The Single-Modal Approach: In this case the Constant Velocity (CV) model is used to model the state evolution. According to the $\mathrm{CV}$ model, matrix $\mathbf{F}$ is given as:

$$
\mathbf{F}=\left[\begin{array}{cccc}
1 & 0 & T_{s} & 0 \\
0 & 1 & 0 & T_{s} \\
0 & 0 & 1 & 0 \\
0 & 0 & 0 & 1
\end{array}\right]
$$

2) Multi-Modal Approach: The multi-modal approach is proposed to provide support when a manoeuvring target is the object of interest. In this case our system is modeled using multiple switching dynamic models. The dynamic models we consider are, the CV model described previously and two coordinated turn models. The model's measurement equation is the same as discussed in Section IV-A, while the evolution of the state in time is modeled with the following nonlinear equation:

$$
\mathbf{x}_{k}=\mathbf{F}\left(\mathbf{r}_{k}\right) \mathbf{x}_{k-1}+\Gamma \mathbf{w}_{k-1}
$$

- where $\mathbf{r}_{k}$ is the regime variable and indicates which model is in use during the sampling period from $\left(t_{k-1}, t_{k}\right]$. The regime variable is modeled as a time homogenous three-state first-order Markov chain with transitional probability matrix,

$$
\pi_{i j} \triangleq P\left\{\mathbf{r}_{k}=j \mid \mathbf{r}_{k-1}=i\right\}
$$

The state transition matrix $\mathbf{F}$, at time $k$ is defined according to the value of the regime variable $\mathbf{r}_{k}\left(\mathbf{F}_{r_{k}}\right)$ and is given as:

- The CV model - As defined in the single-model approach

$$
\mathbf{F}=\left[\begin{array}{cccc}
1 & 0 & T_{s} & 0 \\
0 & 1 & 0 & T_{s} \\
0 & 0 & 1 & 0 \\
0 & 0 & 0 & 1
\end{array}\right]
$$

- First Coordinated Turn model

$$
\mathbf{F}=\left[\begin{array}{cccc}
1 & 0 & \sin \left(w T_{s}\right) / w & \left(\cos \left(w T_{s}\right)-1\right) / w \\
0 & 1 & \left(1-\cos \left(w T_{s}\right)\right) / w & \sin \left(w T_{s}\right) / w \\
0 & 0 & \cos \left(w T_{s}\right) & -\sin \left(w T_{s}\right) \\
0 & 0 & \sin \left(w T_{s}\right) & \cos \left(w T_{s}\right)
\end{array}\right]
$$

- Second Coordinated turn model

$$
\mathbf{F}=\left[\begin{array}{cccc}
1 & 0 & \sin \left(w T_{s}\right) / w T_{s} & \left(\cos \left(w T_{s}\right)-1\right) / w \\
0 & 1 & \left(1-\cos \left(w T_{s}\right)\right) / w & \sin \left(w T_{s}\right) / w \\
0 & 0 & \cos \left(w T_{s}\right) & -\sin \left(w T_{s}\right) \\
0 & 0 & -\sin \left(w T_{s}\right) & \cos \left(w T_{s}\right)
\end{array}\right]
$$

- where $T_{s}$ is the sampling period and,

- where $w$ is the turning rate in $\mathrm{rad} / \mathrm{sec}$

Although the complexity of the system increases with the use of a multi-modal approach, as the state update equation also becomes nonlinear, manoeuvring targets require to be modeled in such a way in order for the system to cope with abrupt changes regarding the amplitude and direction of the velocity vector.

\section{Tracking Algorithms}

This section provides insight regarding the algorithms developed to infer the state of the target at each time step. Specifically, an estimate of the state $\mathbf{x}_{k}$ at time $k$ is produced based on the sequence of measurements $\mathbf{Z}_{k}$ up to that time instance. To calculate a state estimate, the posterior probability density function (pdf) $p\left(\mathbf{x}_{k} \mid \mathbf{Z}_{k}\right)$ should be constructed. After obtaining the posterior pdf $p\left(\mathbf{x}_{k} \mid \mathbf{Z}_{k}\right)$, an estimation of the actual state can be produced, using a certain criterion, like the Minimum Mean Square Error (MMSE). PF algorithms rely upon representing the required pdf with a set of particles and their corresponding weights. Particles are sampled from a proposal distribution and then weighted properly to represent the state's pdf. Let's denote the evolution of the state vector up to time $k$ as $\mathbf{X}_{k}=\left\{\mathbf{x}_{j}: j=1,2, \ldots, k\right\}$. Similar to this, the measurements made available up to time $k$, are denoted as $\mathbf{Z}_{k}=\left\{\mathbf{z}_{j}: j=1,2, \ldots, k\right\}$. The pdf $p\left(\mathbf{x}_{k} \mid \mathbf{Z}_{k}\right)$ is approximated by a set of $N$ particles denoted as $\mathbf{X}_{k}^{i}$ and their corresponding weights, $w_{k}^{i}$. An approximation of the state's pdf at time $k$ is given from the following:

$$
p\left(\mathbf{X}_{k} \mid \mathbf{Z}_{k}\right)=\sum_{i=1}^{N} w_{k}^{i} \delta\left(\mathbf{X}_{k}-\mathbf{X}_{k}^{i}\right)
$$

where $\delta$ is Dirac's delta function.

Particles $\mathbf{X}_{k}^{i}$ are sampled from a proposal distribution $q\left(\mathbf{X}_{k} \mid \mathbf{Z}_{k}\right)$. The importance weight $w_{k}^{i}$ for each particle is computed as follows:

$$
w_{k}^{i} \propto \frac{p\left(\mathbf{X}_{k}^{i} \mid \mathbf{Z}_{k}\right)}{q\left(\mathbf{X}_{k}^{i} \mid \mathbf{Z}_{k}\right)}
$$

PF usually suffer from the degeneracy problem. In practical terms, after a number of iterations all but one particle have negligible weights. Thus, a substantial amount of computation is devoted in updating particles with minimal contribution to the approximation of the pdf. To avoid the effects caused by degeneracy in PF, a measure, called effective sample size $N_{e f f}$, is introduced and defined as follows:

$$
N_{e f f}=\frac{1}{\sum_{i=1}^{N}\left(w_{k}^{i}\right)^{2}}
$$


A resampling step is carried out whenever $N_{e f f}$ is found to be smaller than a pre-defined threshold $N_{t h r}$. Resampling eliminates samples with low importance weights and multiplies samples with high importance weights.

\section{A. Range only Tracking Particle Filter Algorithm ROT-PF}

The algorithm described in this section is intended for a tracking scenario which follows the state-space model analyzed in Section IV-B1. To begin with, we considered that both the state and measurements noise follow known distributions that can be sampled. The transitional prior $p\left(\mathbf{x}_{k} \mid \mathbf{x}_{k-1}\right)$ is chosen as the importance density function to sample particles from. To sample a particle $\mathbf{x}_{k}^{i}$ from $p\left(\mathbf{x}_{k} \mid \mathbf{x}_{k-1}\right)$, a process noise sample $\mathbf{w}_{k-1}^{i}$ is generated from the noise distribution $p_{w}$, and then passed in Eq. 3 to produce $\mathbf{x}_{k}^{i}$. Initial particles (at time $t=0)$ are drawn from a distribution $p\left(x_{0}\right)$ which represents the system's prior knowledge regarding the target's initial state. Upon receiving a new set of measurements the weight for each particle is computed. Because of the transitional prior being the importance density function, Eq.12 simplifies to $\mathbf{w}_{k}^{i} \propto p\left(\mathbf{z}_{k} \mid \mathbf{x}_{k}^{i}\right)$. For this, every particle $\mathbf{x}_{k}^{i}$ is passed through Eq. 2 to produce a predicted observation $\mathbf{z}_{k}^{i}$ which is compared to the real observation and used at the calculation of the likelihood function $p\left(\mathbf{z}_{k} \mid \mathbf{x}_{k}^{i}\right)$. In simple terms, at every time step $k$ the weights are equal to the likelihood of the real-world observation $\mathbf{z}_{k}$, given a realization of the predicted observation $\mathbf{z}_{k}^{i}$ based on the sampled particle $\mathbf{x}_{k}^{i}$. Thus the importance weights for each particle are calculated from the following:

$$
\tilde{w}_{k}^{i}=L\left(\mathbf{z}_{k} \mid x_{k}^{i}\right)=\frac{1}{B} \exp \left(\left(-0.5 *\left(z_{k}-z_{k}^{i}\right) *\left(z_{k}-z_{k}^{i}\right)^{T}\right)\right.
$$

where $B$ is a constant which depends on the parameters of the measurements noise distribution which is considered to be Gaussian. The final step involves resampling, whenever $N_{\text {eff }}$ is found to be smaller than $N_{t h r}$. A single iteration of the ROT-PF algorithm is given in Algorithm 1.

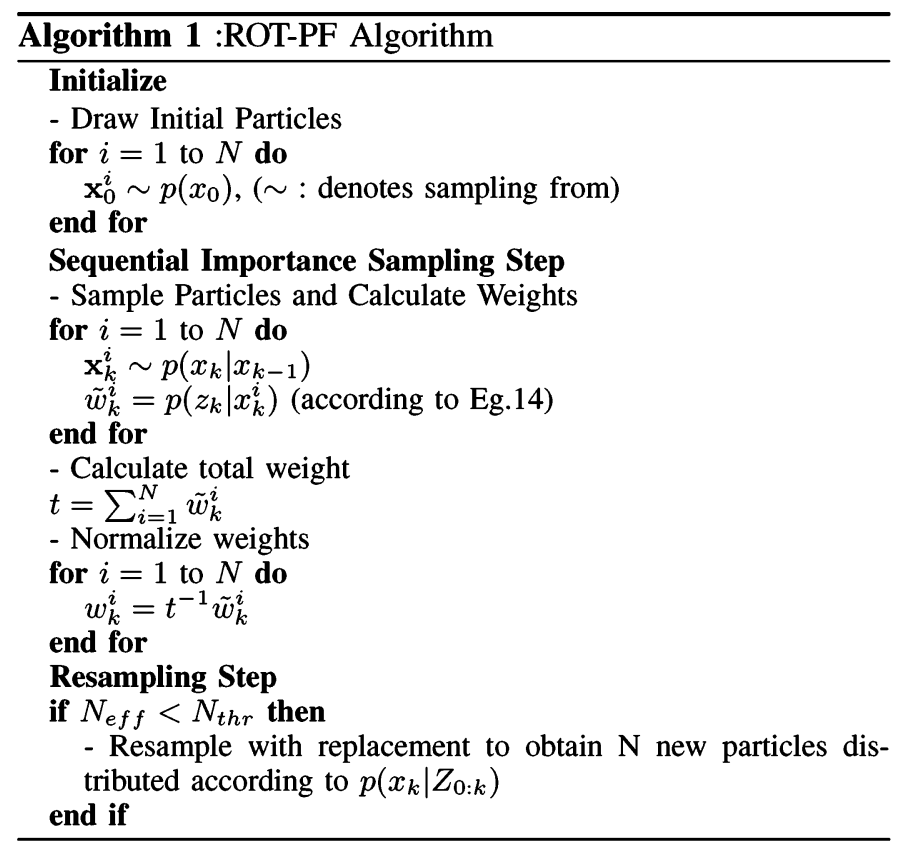

\section{B. Range only Tracking Multiple Model Particle Filter Algo- rithm ROT-MMPF}

To estimate the state vector in the switching dynamical model presented in Section IV-B2, a multiple model PF algorithm is employed. The state vector in the multi-modal case is an augmented state vector which contains both the state $\mathbf{x}_{k}$ and the regime variable $\mathbf{r}_{k}$. The augmented state vector is denoted as, $\mathbf{y}_{k}=\left[\mathbf{x}_{k} \mathbf{r}_{k}\right]$. Initial particles are drawn from two distributions $p\left(\mathbf{r}_{0}\right)$ and $p\left(\mathbf{x}_{0}\right)$ which represent the system's initial knowledge regarding the system's state. Particles for the state $\mathbf{x}_{k}$ are sampled from the transitional prior, in the same way as in the ROT-PF case, while particles for the regime variable are sampled according to the transitional probability matrix $\Pi=\left[\pi_{m n}\right]$. The rule that is followed is that, if $r_{k-1}^{i}=m$, then $r_{k}^{i}$ should be set to $n$ with probability $\pi_{m n}$. Similar to the ROT-PF algorithm, when a new measurement vector becomes available the corresponding weights for each particle $\mathbf{x}_{k}^{i}$ are computed using the likelihood function $p\left(\mathbf{z}_{k} \mid \mathbf{x}_{k}^{i}, \mathbf{r}_{k}^{i}\right)$.

The final step of the ROT-MMPF algorithm includes the resampling step whenever this is necessary. An iteration of the ROT-MMPF algorithm is given in Algorithm 2.

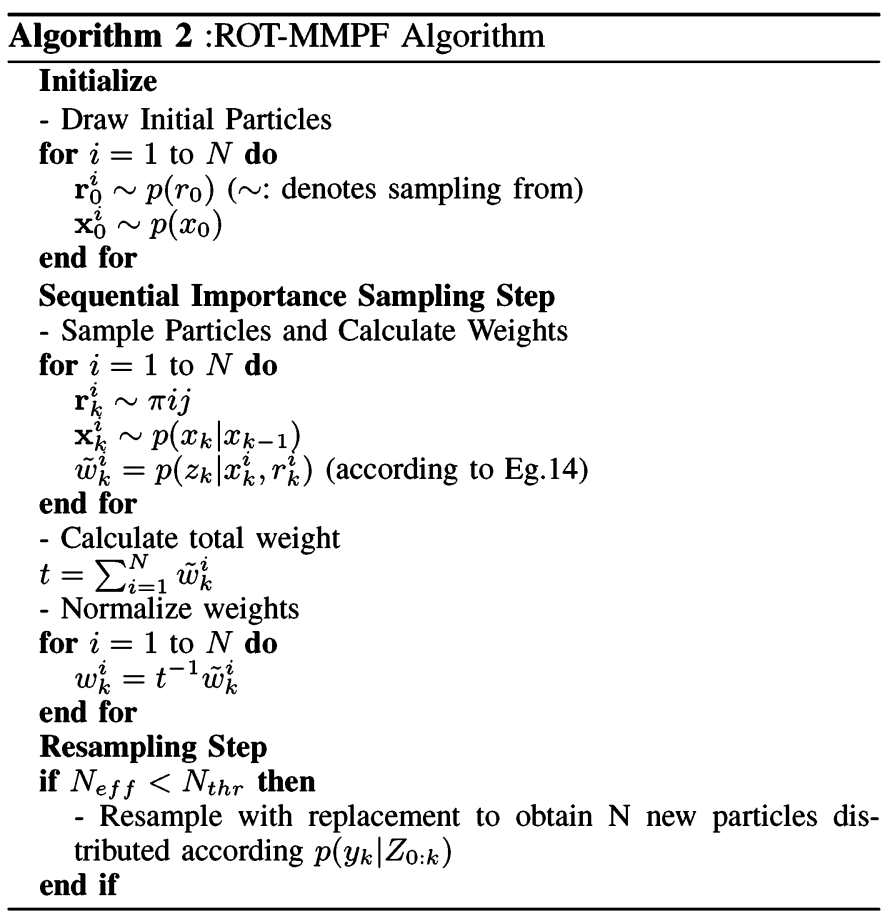

\section{SimUlation - RESUlTS}

Both the ROT-PF and the ROT-MMPF algorithms were simulated in different conditions to evaluate their performance in terms of tracking accuracy and robustness. In the simulations environment considered, four anchor nodes are deployed in known locations to provide the system with the range observations, while a single ground mobile target is the object of interest. To acquire a range estimate from each of the 
anchor nodes, the target should be within the communication reach of the anchor nodes. In practice, processing the ranging measurements and inferring an estimation regarding the target's position requires a certain amount of time to be devoted to that process. By reducing the time that the system requires to infer an estimate, faster targets can be considered for tracking. For the technology considered, the nodes are able to communice within a range of $250 \mathrm{~m}$. As fas as the the object's maximum velocity is concerned, the upper bound depends on the required time to gather and fuse the range estimates from the anchor nodes and also the execution time of the algorithm. However for the purposes of simulation, the ranging estimates are considered to be instantly acquired.

\section{A. Evaluating the ROT-PF algorithm}

1) First Scenario: This section presents simulation results of the ROT-PF algorithm. A wireless network consisting of four anchor nodes is considered to be deployed. The coordinates of the anchor nodes are, $s_{1}=[40 \mathrm{~m} 60 \mathrm{~m}], s_{2}=$ $[40 \mathrm{~m} 140 \mathrm{~m}], s_{3}=[100 \mathrm{~m} 60 \mathrm{~m}], s_{4}=[100 \mathrm{~m} 140 \mathrm{~m}]$. The state vector of the target evolves in time as defined in Eq.5, while the measurements are associated to the target's state according to Eq.2. The sampling period is set to $T_{s}=1 \mathrm{sec}$ and the system evolves for $T=50 \mathrm{sec}$. In the implementation of the ROT-PF tracking algorithm $N=500$ particles were used. The measurements and state noise sources are considered to follow zero mean Gaussian distributions. Specifically:

$$
\begin{aligned}
\mathbf{w}_{k} & \sim \mathcal{N}\left(0,0.3 \mathbf{I}_{2}\right) \\
\mathbf{v}_{k} & \sim \mathcal{N}\left(0,0.4 \mathbf{I}_{4}\right)
\end{aligned}
$$

where $\mathbf{I}$ is the identity matrix. The target's initial state is

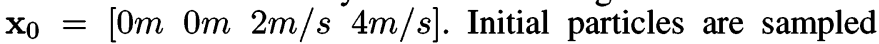
from a Gaussian distribution with zero mean and covariance matrix $\mathbf{S}_{0}=\mathbf{I}_{4}$. The above system was simulated for a total of $L=100$ times and the root mean square error (RMSE) for the position was calculated at each execution. The RMSE for position is defined in Eq.15 In Figure 2 and Figure 3 results from executing a single run of the above system are presented.

$$
\mathbf{R M S E}=\sqrt{\frac{1}{T} \sum_{t=1}^{T}\left(x_{t}-x_{t, e s t}\right)^{2}+\left(y_{t}-y_{t, e s t}\right)^{2}}
$$

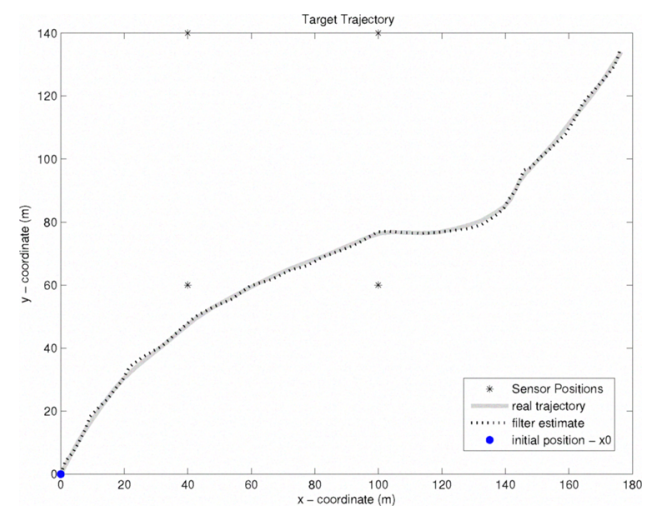

Fig. 2. True and Filtered target trajectory - ROT-PF Scenario 1

The RMSE for the exemplar run was calculated, RMSE = $0.9056 \mathrm{~m}$. The RMSE for the total of 100 runs is illustrated

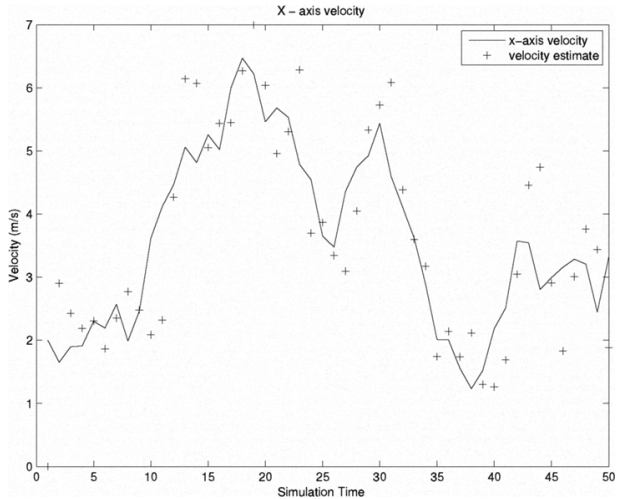

(a) $\mathrm{x}$-axis velocity estimation

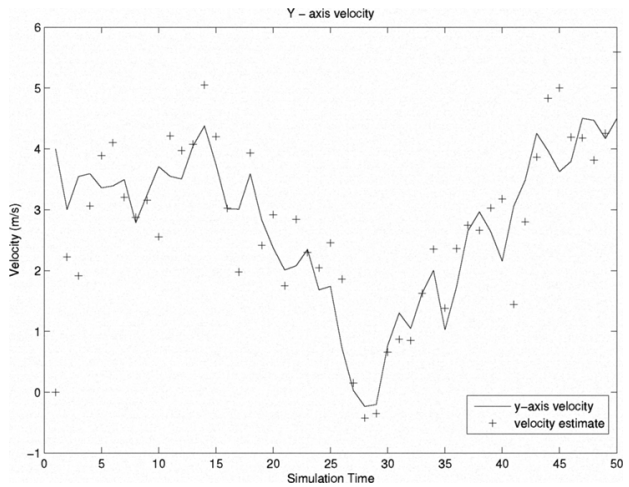

(b) y-axis velocity estimation

Fig. 3. Two-axis velocity estimation - ROT-PF Scenario 1

in Figure 4(a). Only in four runs the RMSE increases into unsatisfactory levels RMSE $>100 \mathrm{~m}$. In the majority of the executions, the RMSE remains low (RMSE $<20 m$ ) which reveals the robustness of the tracking system. The RMSE error can be further decreased if the uncertainty of the system regarding the target's initial state is reduced. In Figure 4(b) the same system is simulated for 100 runs, only this time initial particles are drawn from a Gaussian distribution, with the same covariance matrix $\mathbf{S}_{0}$ but with mean $\mu_{0}=x_{0}+\mathcal{N}\left(0,1.5^{2}\right)$. The substantially improved RMSE is due to the fact that the system has better knowledge over the target's initial condition.

2) Second scenario: A scenario of high initial uncertainty and heavy noised is considered. Figure 5 depicts the ability of the ROT-PF algorithm to perform accurately under such conditions. The anchor nodes are positioned in the same coordinates as previously also the same number $N=500$

\begin{tabular}{|c|c|}
\hline \multicolumn{2}{|r|}{ Parameters } \\
\hline $\mathbf{w}_{k}$ & $\sim \mathcal{N}\left(0,2 \mathbf{I}_{2}\right)$ \\
\hline $\mathbf{v}_{k}$ & $\sim \mathcal{N}\left(0,2 \mathbf{I}_{4}\right)$ \\
\hline$\mu_{0}$ & {$\left[\begin{array}{llll}0 & 0 & 0 & 0\end{array}\right]$} \\
\hline$P_{0}$ & I \\
\hline$x_{0}$ & {$[2 \mathrm{~m} 1 \mathrm{~m} 2 \mathrm{~m} / \mathrm{s} 5 \mathrm{~m} / \mathrm{s}]$} \\
\hline$T$ & $100 \mathrm{sec}$ \\
\hline
\end{tabular}
of particles was used. The rest of the parameters for this case are given in the following table: 


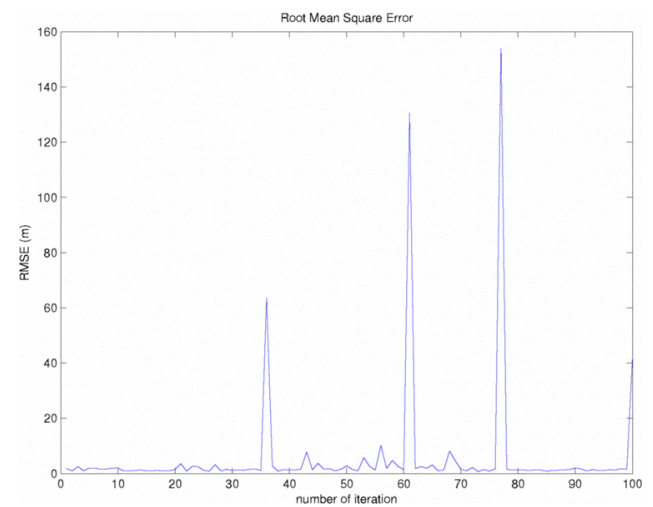

(a) RMSE for 100 simulation runs $-\mu_{0}=0$

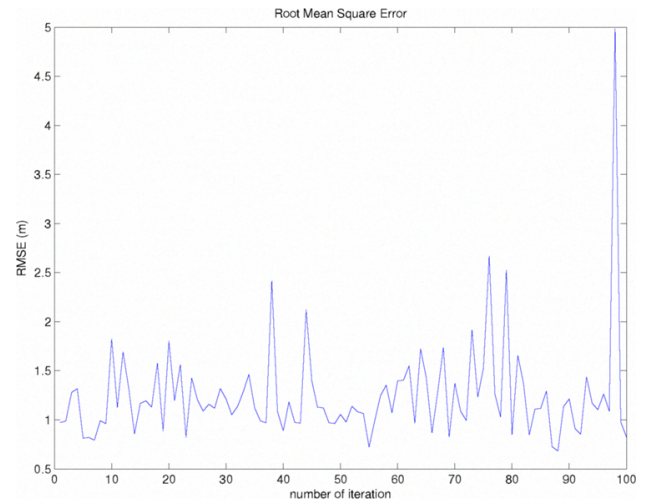

(b) RMSE for 100 simulation runs $-\mu_{0}=x_{0}+\mathcal{N}\left(0,1.5^{2}\right)$

Fig. 4. RMSE for 100 simulation runs of the ROT-PF - Scenario 1

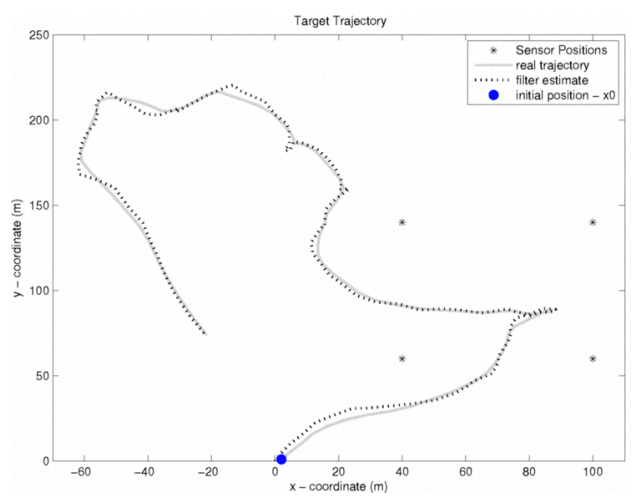

Fig. 5. True and Filtered Trajectory - ROT-PF Scenario $2-\mathbf{R M S E}=2.22 \mathrm{~m}$

\section{B. Evaluating the ROT-MMPF algorithm}

In this section results from simulating two different scenarios with the ROT-MMPF algorithm are provided. The aim of this algorithm is to enable our system to keep track of manoeuvring targets which is usually the case in real-world applications. The three-state model we employ in this case is sufficient to provide adequate support for manoeuvring targets.
1) First Scenario: Four anchor nodes are considered, positioned at the following coordinates $s_{1}=[0 \mathrm{~m} 0 \mathrm{~m}], s_{2}=$ $[200 \mathrm{~m} 0 \mathrm{~m}], s_{3}=[0 \mathrm{~m}-80 \mathrm{~m}], s_{4}=[200 \mathrm{~m}-80 \mathrm{~m}]$. The simulation time is set to $T=80 \mathrm{sec}$, the sampling period to $T_{s}=1 \mathrm{sec}$ and the turning rate to $w=\pi / 4 \mathrm{rad} / \mathrm{s}$. The number of particles used in the implementation of the algorithm is $N=1000$. The noise sources are modeled as zero mean additive Gaussian noise with distributions:

$$
\begin{aligned}
\mathbf{w}_{k} & \sim \mathcal{N}\left(0,0.1 \mathbf{I}_{2}\right) \\
\mathbf{v}_{k} & \sim \mathcal{N}\left(0, \mathbf{I}_{4}\right)
\end{aligned}
$$

The regime variable is defined as a first order homogenous Markov chain with transition probability $m=0.5$ thus the transition probability matrix is,

$$
\mathbf{P}\left(r_{t} \mid r_{t-1}\right)=\left[\begin{array}{ccc}
0.5 & 0.25 & 0.25 \\
0.25 & 0.5 & 0.25 \\
0.25 & 0.25 & 0.5
\end{array}\right]
$$

The target's actual initial state vector is $\mathbf{x}_{0}=$ $[0 \mathrm{~m} 0 \mathrm{~m} 0.03 \mathrm{~m} / \mathrm{s} 0.04 \mathrm{~m} / \mathrm{s}]$. Initial particles for the regime variable $\mathbf{r}_{\mathbf{t}}$ are sampled with equal probability $\mathbf{P}_{\mathbf{0}}=$ $\left[\begin{array}{lll}0.333 & 0.333 & 0.333\end{array}\right]$ (which is $=1 / 3$ because there are three possible regimes), while initial particles for the state $\mathbf{x}_{k}$ are drawn from a Gaussian distribution with zero mean and covariance matrix:

$$
\mathbf{S}_{0}=\left[\begin{array}{cccc}
0.01 & 0 & 0 & 0 \\
0 & 0.01 & 0 & 0 \\
0 & 0 & 0.001 & 0 \\
0 & 0 & 0 & 0.001
\end{array}\right]
$$

The results from a single run of the above scenario are illustrated in Figure 6 for the target's trajectory and in Figure 7 for the target's two-axis velocity. The RMSE was calculated

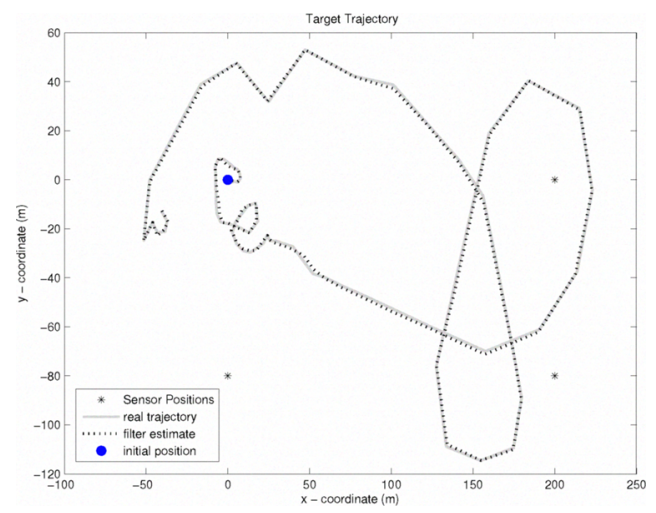

Fig. 6. True and Filtered trajectory - ROT-MMPF Scenario 1

RMSE $=9.2992 m$.

2) Second Scenario: For this scenario the anchor nodes are positioned at $s_{1}=[10 \mathrm{~m} 0 \mathrm{~m}], s_{2}=[50 \mathrm{~m} 0 \mathrm{~m}], s_{3}=$ $[10 \mathrm{~m} 25 \mathrm{~m}], s_{4}=[50 \mathrm{~m} 25 \mathrm{~m}]$. The duration of the simulation is $T=80 \mathrm{sec}$, the sampling period is $T_{s}=1 \mathrm{sec}$ and the turning rate $w=\pi / 3 \mathrm{rad} / \mathrm{s}$. The number of particles used in this scenario is the same as previously $N=1000$. The noise sources follow identical Gaussian distributions with zero mean and covariance $\sigma_{w}=\sigma_{v}=0.1$. The regime variable is defined in a similar way as in the previous scenario, thus the same transition matrix applies here and the target's initial state 


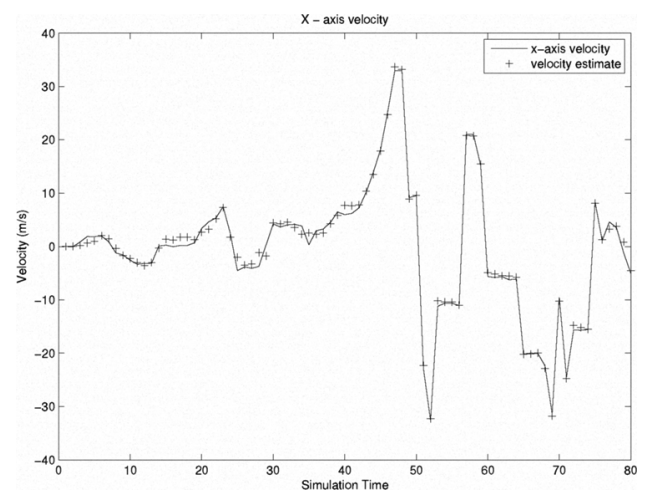

(a) $\mathrm{x}$-axis velocity estimation

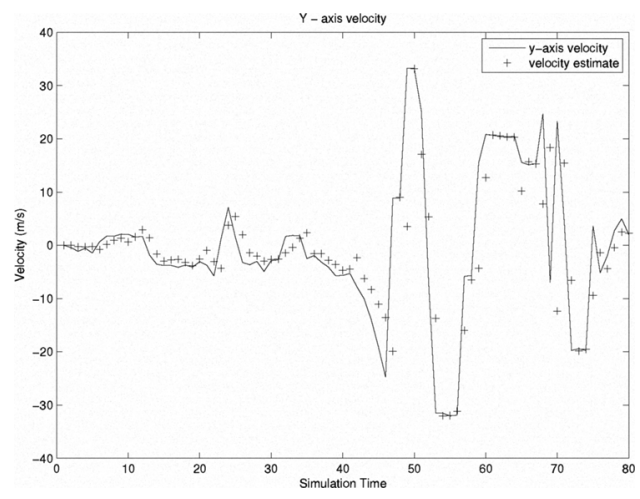

(b) y-axis velocity estimation

Fig. 7. Two-axis velocity estimation - ROT-MMPF Scenario 1

is $x_{0}=[0 \mathrm{~m} 0 \mathrm{~m} 3 \mathrm{~m} / \mathrm{s} 4 \mathrm{~m} / \mathrm{s}]$. Finally, initial particles for the state vector $\mathbf{x}_{k}$ are sampled from a zero mean Gaussian distribution with covariance matrix;

$$
\mathbf{S}_{0}=\left[\begin{array}{cccc}
0.1 & 0 & 0 & 0 \\
0 & 0.1 & 0 & 0 \\
0 & 0 & 0.1 & 0 \\
0 & 0 & 0 & 0.1
\end{array}\right]
$$

Trajectory results from the simulation of this scenario are illustrated in Figure 8, while velocity estimation is depicted in Figure 9.

This scenario is also simulated for a total of 100 executions and the RMSE is calculated in every run. In 61 of the executions the RMSE remains below $100 \mathrm{~m}$, while 78 executions demonstrate RMSE below $1000 \mathrm{~m}$. However in some occasions the RMSE increases in high levels RMSE $>10^{3} \mathrm{~m}$ where tracking is considered failed. Similar to the ROT-PF algorithm, the RMSE in the ROT-MMPF algorithm can be reduced by minimizing the uncertainty of the system regarding the target's initial state. The RMSE from a similar scenario, but with initial particles drawn from a Gaussian distribution with mean $\mu_{0}=\mathbf{x}_{0}+\mathcal{N}(0,1)$ and the same covariance matrix as previously. The RMSE from 100 executions of this system is presented in Figure 10(b). In 98 of the executions the RMSE is calculated to be smaller than $1000 \mathrm{~m}$ and in 92 execution the RMSE is smaller than $100 \mathrm{~m}$.

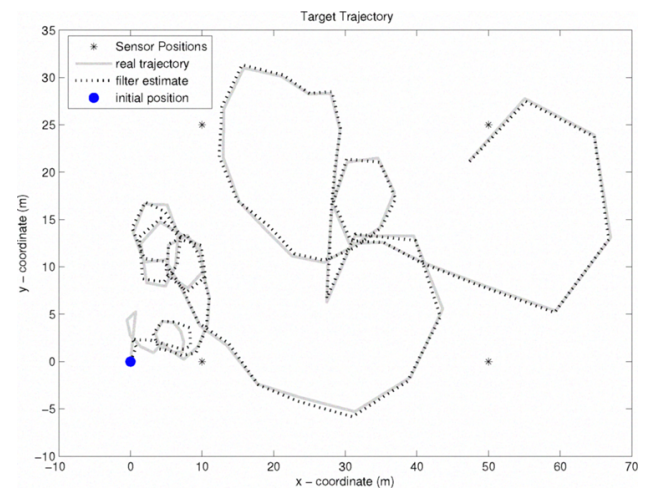

Fig. 8. True and filtered trajectory - ROT-MMPF Scenario 2

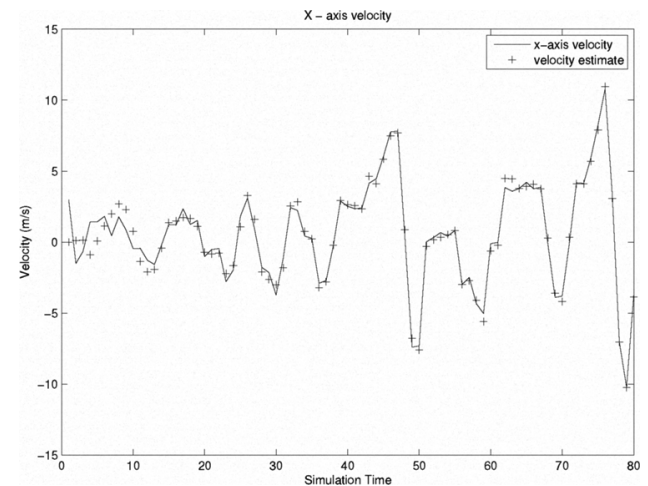

(a) $\mathrm{x}$-axis velocity estimation

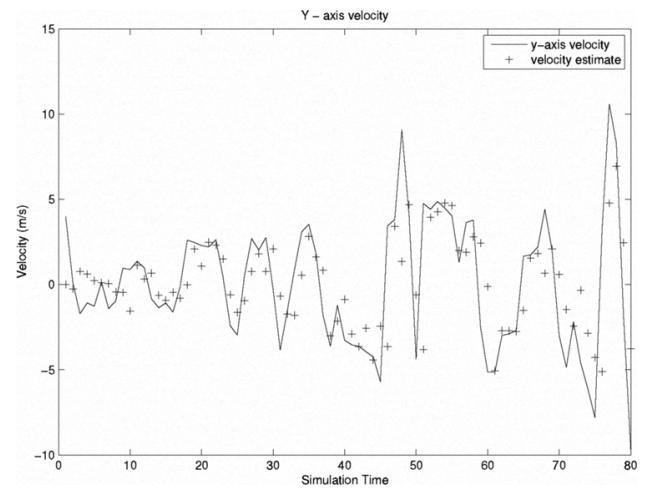

(b) y-axis velocity estimation

Fig. 9. Two-axis velocity estimation - ROT-MMPF Scenario 2

\section{CONCLUSIONS AND FUTURE WORK}

The prominent outcomes obtained from simulating the system for a number of different scenarios indicate that accurate tracking of manoeuvring ground targets can be achieved with range-only measurements using WSNs. In the majority of the simulations the RMSE is small proving the accuracy of the tracking algorithm. Simulation results also indicate that a factor which affects the accuracy of our system is the system's knowledge regarding the object's initial state. In 


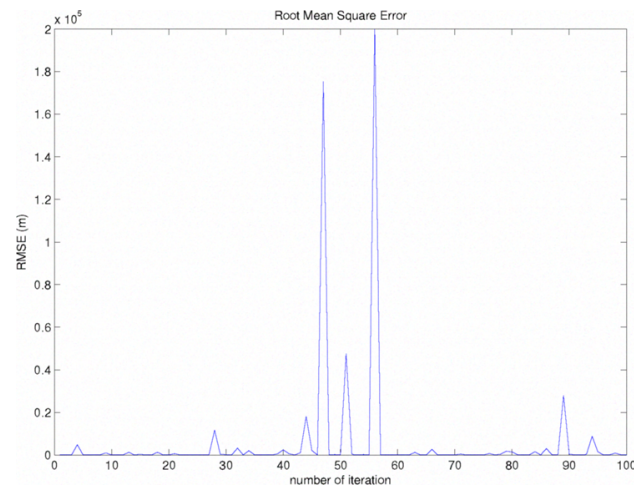

(a) RMSE for 100 simulation runs $-\mu_{0}=0$

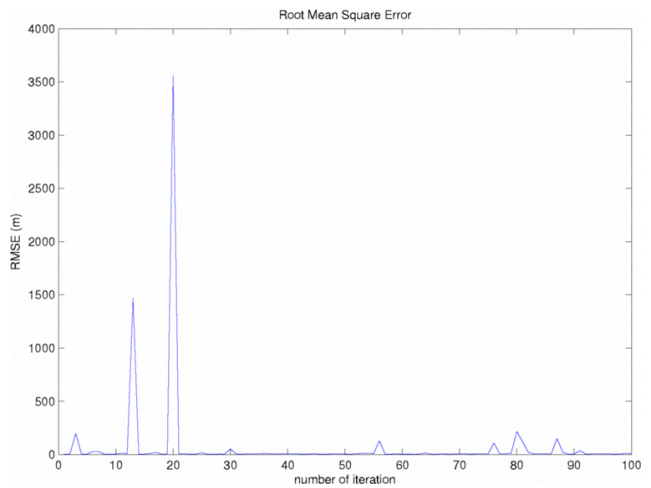

(b) RMSE for 100 simulation runs $-\mu_{0}=x_{0}+\mathcal{N}(0,1)$

Fig. 10. RMSE for 100 simulation runs of the ROT-MMPF

addition, accuracy can be amended by employing a bigger number of particles, subsequently increasing the computational demand. The number of required particles in the proposed system is kept small compared to other approaches. Different to other approaches the proposed system does not require any additional type of measurements in order to track manoeuvring targets.

As hardware becomes smaller in size and easier to obtain, more challenges regarding commercial use of WSNs will arise. Our future plans comprise of conducting research along the direction of implementing a real-world, online tracking system. Specifically, our own laboratory experiments have proved that ranging with up to $1 \mathrm{~m}$ accuracy between two nodes can be achieved with ToF techniques. Since accurate ranging is feasible, integrating the algorithms and implementing the tracking operation on WSNs hardware will be the evolution of the work presented in this paper.

\section{REFERENCES}

[1] D. Culler, D. Estrin, and M. Srivastava, "Guest editors' introduction Overview of sensor networks," IEEE Computer's Society, vol. 37, pp. 41-49, 2004.

[2] K. Whitehouse, C. Karlof, and D. Culler, "A practical evaluation of radio signal strength for ranging-based localization," SIGMOBILE Mob. Comput. Commun. Rev., vol. 11, no. 1, pp. 41-52, 2007.

[3] S. Lanzisera, D. T. Lin, and K. S. Pister, "Rf time of flight ranging for wireless sensor networks localization," in Proc. of WISES, Jun. 2006.
[4] P. Bahl and V. Padmanabhan, "Radar: an in-building rf-based user location and tracking system," in IEEE Infocom, Mar. 2000.

[5] R. Brooks, P. Ramanathan, and A. Sayeed, "Distributed target classification and tracking in sensor networks," Proceedings of the IEEE, vol. 91, no. 8, pp. 1163-1171, Aug. 2003.

[6] A. Aroraet. al, "A line in the sand: a wireless sensor network for target detection, classification, and tracking," Comput. Netw., vol. 46, no. 5, 2004.

[7] T. He, S. Krishnamurthy, J. A. Stankovic, T. Abdelzaher, L. Luo, R. Stoleru, T. Yan, L. Gu, J. Hui, and B. Krogh, "Energy-efficient surveillance system using wireless sensor networks," in Proc. of MobiSys, Jun. 2004.

[8] T. He, P. Vicaire, T. Yan, L. Luo, L. Gu, G. Zhou, R. Stoleru, Q. Cao, J. Stankovic, and T. Abdelzaher, "Achieving real-time target tracking usingwireless sensor networks," in Proc. of IEEE RTAS, Apr. 2006.

[9] T. Bokareva, W. Hu, S. Kanhere, B. Ristic, N. Gordon, T. Bessell, M. Rutten, and S. Jha, "Wireless sensor networks for battlefield surveillance," in Proc. of $L W C$, Oct. 2006.

[10] P. Chenet. al, "Experiments in instrumenting wireless sensor networks for real-time surveillance," in Proc. of ICRA, May. 2006.

[11] S. Oh, S. Sastry, and L. Schenato, "A hierarchical multiple-target tracking algorithm for sensor networks," in Proc. ICRA, Apr. 2005.

[12] F. Gustafsson and F. Gunnarsson, "Mobile positioning using wireless networks: possibilities and fundamental limitations based on available wireless network measurements," IEEE Signal Processing Magazine, vol. 22 , no. 4, pp. 41-53, July 2005.

[13] J. Liu, J. Reich, and F. Zhao, "Collaborative in-network processing for target tracking," EURASIP J. Appl. Signal Process., vol. 2003, no. 1, 2003.

[14] F. Zhao, J. Liu, J. Liu, L. Guibas, and J. Reich, "Collaborative signal and information processing: an information-directed approach," Proc of the IEEE, vol. 91, no. 8, Aug. 2003.

[15] J. Singh, U. Madhow, R. Kumar, S. Suri, and R. Cagley, "Tracking multiple targets using binary proximity sensors," in Proc. of IPSN, Apr. 2007.

[16] N. Shrivastava, R. M. U. Madhow, and S. Suri, "Target tracking with binary proximity sensors: fundamental limits, minimal descriptions, and algorithms," in Proc. of SenSys, Oct-Nov. 2006.

[17] Z. Zhong, T. Zhu, D. Wang, and T. He, "Tracking with unreliable node sequences," in IEEE Infocom, Apr. 2009.

[18] Y. Bar-Shalom and X.-R. Li, Estimation with Applications to Tracking and Navigation. John Wiley \& Sons, Inc., 2001.

[19] B. Kusy, A. Ledeczi, and X. Koutsoukos, "Tracking mobile nodes using rf doppler shifts," in Proc. of SenSys, Oct-Nov. 2007.

[20] M. Arulampalam, S. Maskell, N. Gordon, and T. Clapp, "A tutorial on particle filters for online nonlinear/non-gaussian bayesian tracking," IEEE Trans. on Signal Processing, Feb 2002.

[21] A. Doucet, N. Gordon, and V. Krishnamurthy, "Particle filters for state estimation of jump markov linear systems," IEEE Trans. on Signal Processing, vol. 49, no. 3, Mar 2001.

[22] B. Ristic, S. Arulampalam, and N. Gordon, Beyond the Kalman Filter - Particle Filters For Tracking Applications. Artech House Publishers, 2004.

[23] M. Coates, "Distributed particle filters for sensor networks," in Proc. of IPSN, Apr. 2004.

[24] M. Coates and G. Ing, "Sensor network particle filters: motes as particles," in Proc. of SSPW, Jul. 2005.

[25] M. Borkar, V. Cevher, and J. McClellan, "Estimating target state distributions in a distributed sensor network using a monte-carlo approach," in Proc. of WMLSP, Sep. 2005.

[26] X. Sheng and Y. H. Hu, "Sequential acoustic energy based source localization using particle filter in a distributed sensor network," in Proc. of ICASSP, May. 2004.

[27] H. Ma and B.-H. Ng, "Distributive target tracking in wireless sensor networks under measurement origin uncertainty," in Proc. of ISSNIP, Dec. 2007.

[28] Y. K. Lee, E. H. Kwon, and J. S, "'self location estimation scheme using roa in wireless sensor networks"," in Proc. of EUC Workshops, Dec. 2005. 